\title{
Characteristics of the L138ins (p.Leu138dup) mutation in Russian cystic fibrosis patients
}

\section{Nika V Petrova}

Research Centre for Medical Genetics, Moscow

(D) https://orcid.org/0000-0001-5933-6594

\section{Nataliya Y Kashirskaya}

Research Centre for Medical Genetics, Moscow

(D) https://orcid.org/0000-0003-0503-6371

Corresponding author: kashirskayanj@mail.ru

Tatyana A Vasilyeva

Research Center for Medical Genetics, Moscow

(iD) https://orcid.org/0000-0002-6744-0567

\section{Elenai I Kondratyeva}

Research Center for Medical Genetics, Moscow

(iD) https://orcid.org/0000-0001-6395-0407

\section{Andrey V Marakhonov}

Research Center for Medical Genetics, Moscow

(D) https://orcid.org/0000-0002-0972-5118

\section{Milan Macek Jr}

$2^{\text {nd }}$ Faculty of Medicine, Charles University,

Prague; Motol University Hospital, Prague

(iD) https://orcid.org/0000-0002-5173-5280

\section{Evgeny K Ginter}

Research Center for Medical Genetics, Moscow

(iD) https://orcid.org/0000-0001-6920-6726

\section{Sergey I Kutsev}

Research Center for Medical Genetics, Moscow

(iD) https://orcid.org/0000-0002-3133-8018

\section{Rena A Zinchenko}

Research Center for Medical Genetics, Moscow; National Institute of Public Health named after N.A. Semashko

(D) https://orcid.org/0000-0003-3586-3458

DOI: https://doi.org/10.20883/medical.383

Keywords: cystic fibrosis, haplotype, L138ins (c.411_412insCTA, p.Leu138dup) mutation

Published: 2020-01-30

\begin{abstract}
How to Cite: Petrova NV, Kashirskaya NY, Vasilyeva TA, Kondratyeva El, Marakhonov AV, Macek Jr M, Ginter EK, Kutsev SI, Zinchenko RA. Characteristics of the L138ins (p.Leu138dup) mutation in Russian cystic fibrosis patients. JMS [Internet]. 2020 Mar 31;89(1):e383. doi: 10.20883/medical.383
\end{abstract}

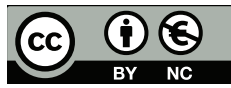

(c) 2020 by the author(s). This is an open access article distributed under the terms and conditions of the Creative Commons Attribution (CC BY-NC) licencse. Published by Poznan University of Medical Sciences

\begin{abstract}
The L138ins mutation, found in Russian cystic fibrosis (CF) patients, is a duplication of three nucleotides (CTA) in exon 4 of the CFTR gene and is categorised as a small in-frame insertion/deletion. As a result, the CFTR protein molecule elongates by one amino acid residue, leucine, at position 138 (codon 138 (CTA)). In accordance with the new nomenclature, it should be called c.411_412insCTA (p.Leu138dup). The c.411_412insCTA (p.Leu138dup, L138ins) mutation is found in CF patients of Slavic origin (Russians, Ukrainians) and has been linked to a single haplotype of the intragenic DNA markers IVS1CA-IVS6aGATT-IVS8CA-IVS17bCA - 22-716-13.
\end{abstract}

\section{Introduction}

Cystic fibrosis (CF; OMIM \# 219700) is an autosomal recessive disease caused by a mutation in the CFTR gene (OMIM * 602421), characterised by a variable clinical picture ranging from a rela- tively mild disease course with monosymptomatic manifestations to severe multiorgan lesions [1]. The prevalence of CF in European countries is about 1 in 2500-4500 newborns and in the Russian Federation, it is 1 in 10,000 newborns [1]. The 
spectrum and frequencies of CFTR mutations vary widely among different populations and ethnic groups.

The spectrum of CFTR mutations specific to Russian CF patients has been recently identified. Moreover, the creation of the Russian Cystic Fibrosis Patient Registry (RCF-PR) and the provisions of the National Consensus documents on CF care have made it possible to combine data from clinical trials and researches conducted in different centres to better clarify the frequency of mutations both in the Russian Federation itself and in specific regions within the Federation $[2,3]$. According to the RCF-PR 2016, the ten most common mutations (frequency) are: F508del (52.06\%), CFTRdele2.3 (5.71\%), E92K (2.67\%), 2143delT (2.06\%), 3849+10kbC>T (2.04\%), 2184insA (1.87\%), W1282X (1.82\%), N1303K (1.47\%), 1677delTA (1.44\%), and G542X (1.35\%). Mutation c.411_412insCTA (p.Leu138dup, L138ins) can be considered common in Russian patients since its frequency exceeds $1 \%(1.15 \%)$ of the total number of identified mutant alleles (ranging from $0.29-2.89 \%$ across different regions) $[2,3]$. The frequency of this mutation in Europe has not been determined due to the rarity of this particular pathological allele in European populations [1]. Indeed, the L138ins mutation is not included in the panel of routinely analysed CFTR mutations in European countries. The purpose of this study is to describe the genotypic features of the L138ins mutation in Russian CF patients.

\section{Material and Methods}

DNA was isolated from whole blood samples of CF patients using a DNA extraction kit (Promega, USA). Molecular genetic testing for the c.411_412insCTA (p.Leu138dup, L138ins) mutation was performed using amplified fragment length polymorphism (AFLP) analysis as part of the testing for frequent CFTR mutations in 1700 CF patients and was conducted in the Laboratory of Genetic Epidemiology at the Research Centre for Medical Genetics, Moscow [4]. Sanger sequencing to confirm the c.411_412insCTA (p.Leu138dup, L138ins) mutation presence was performed in $37 \mathrm{CF}$ patients. Analysis of DNA markers IVS1CA, IVS6aGATT, IVS8CA, and IVS17bCA was performed in 24 CF patients carry- ing the c.411_412insCTA (p.Leu138dup, L138ins) mutation and their parents using a previously described procedure [5]. This research project was approved by the Ethics committee of the Research Center for Medical Genetics (Moscow). CF patients, or their fiduciaries, provided written informed consent.

\section{Results and discussion}

In 2000, while testing for the $621+1 \mathrm{G}>\mathrm{T}$ mutation in a Russian CF patient using RFLP test, the abnormal mobility of the exon 4 fragment of the CFTR gene was first observed (Figure 1). Sequencing confirmed the presence of a CFTR mutation (L138ins) not previously found in the Russian population (Figure 2). By 2006, the L138ins mutation was detected in six unrelated CF patients from Moscow and the Moscow region [4]. Subsequently, the L138ins mutation was also identified in two patients from the Krasnodar region [6]. Currently, the L138ins mutation is included in the panel of common CFTR mutations routinely tested for in the Russian population [3].

Analysis of the chromatogram (Figure 1) shows that the initial sequence of 5 '-CACTGCTCCTACACCCAGCC is changed to $5^{\prime}$-CACTGCTCCTACTACACCCAGCC. Formally, four dif-

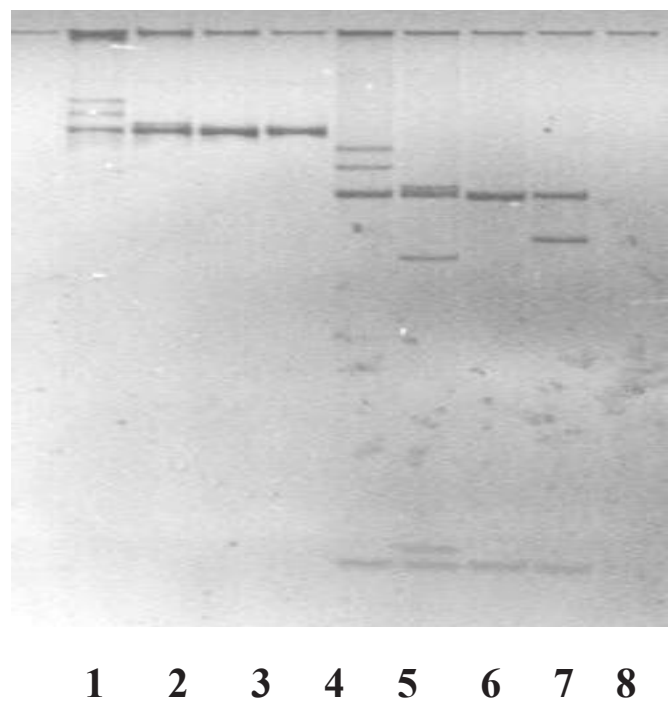

Figure 1. Detection of mutations in exon 4 of the CFTR gene in patients with CF. Lanes 1-4 are amplification products of exon 4: lanes 1 and 2 are samples with abnormal mobility of amplified fragments. Lanes 5 and 6 are restriction products of amplicons 1-4 using Tru1। endonuclease: 5 - L138insA / normal; 6 - 604insA / normal; 7 - normal / normal; $8-621+1 \mathrm{G}>\mathrm{T}$ / normal 


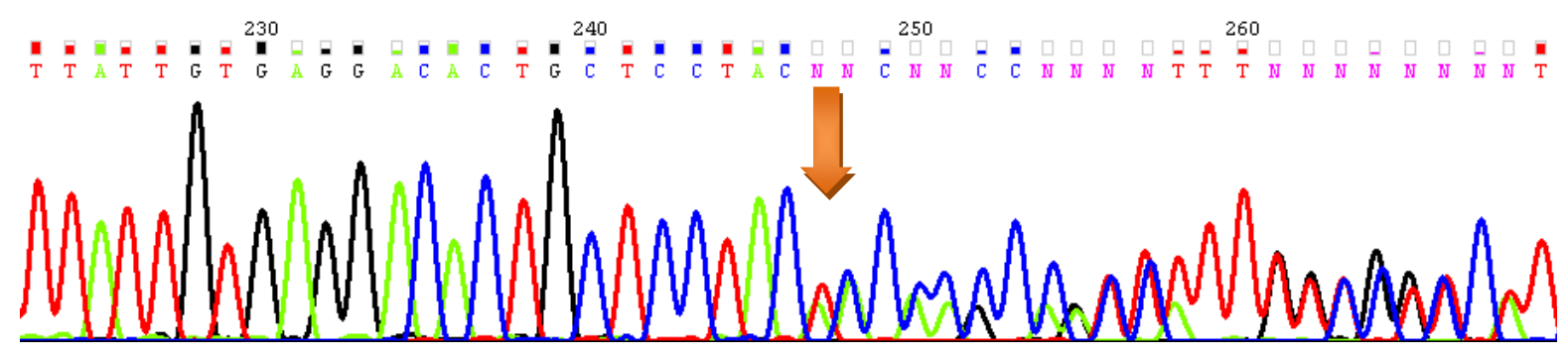

b) from the reverse primer

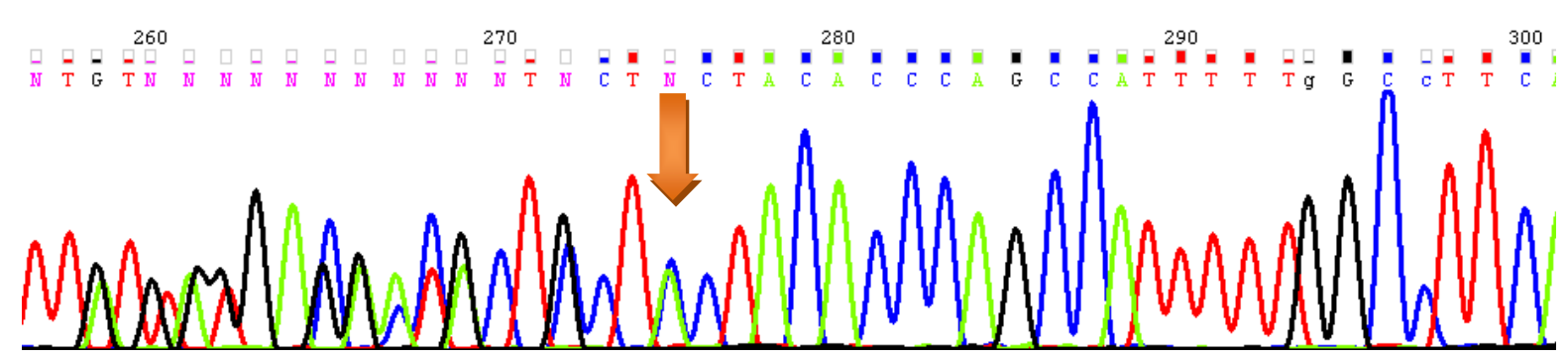

Figure 2. Chromatogram of sequencing results of DNA fragment containing exon 4 of the CFTR gene with the L138ins (p.Leu138dup) mutation

ferent events can lead to such a rearrangement (Table 1): insertion of the CTA triplet between 411 and 412 (1) or 414 and 415 (4) positions of the coding sequence; a TAC insertion between 412 and 413 (2) and an ACT insertion between 413 and 414 (3) positions. Any of these rearrangements will lead to duplication of the CTA codon without changing the reading frame, leading to duplication of leucine at position 138. According to the current nomenclature, the L138ins mutation should be designated as c.411_412insCTA (p.Leu138dup).

This mutation is located in the second membrane penetrating motif of the first transmem- brane domain (MSD1) involved in the formation of the pore of the CFTR chloride channel. The likely consequence of this mutation is the impairment of the conductive properties of the chloride channel. The L138ins mutation was first described by Dörk et al. in 1996 in a 34-year-old patient with a congenital bilateral absence of the vas deferens (CBAVD). The patient was pancreatic sufficient, without lung lesions, a sweat chloride level of $53 \mathrm{mmol} / \mathrm{l}$, with the $5 \mathrm{~T}$ variant in the second allele [7]. The CFTR1 database [8] describes two insertions of three nucleotides in the region under consideration: in one case the mutation, using the legacy nomenclature referred to as

Table 1. Mean values of clinical and functional indices in CF patients with different genotypes

\begin{tabular}{lccc} 
& L138dup/F508del & 3849+10kbC>T/F508del & F508del/F508del \\
\hline Sweat chloride $(\mathrm{mmol} / \mathrm{l})$ & $86.99 \pm 17.02$ & $78.29 \pm 24.29$ & $103.49 \pm 22.89$ \\
\hline Age at diagnosis $(\mathrm{yrs})$ & $6.71 \pm 9.73$ & $14.34 \pm 8.89$ & $1.99 \pm 0.16$ \\
\hline $\mathrm{PI}(\%$ of patients) & 19.1 & 33.3 & 94.1 \\
\hline BMI $\left(\mathrm{kg} / \mathrm{m}^{2}\right)$ & $21.87 \pm 2.58$ & $18.84 \pm 2.73$ & $18.64 \pm 2.46$ \\
\hline FEV1 $(\%)$ & $77.56 \pm 29.43$ & $54.52 \pm 21.88$ & $72.34 \pm 27.21$ \\
\hline Chronic $P$. aeruginosa & $12.5 \%$ & $62.5 \%$ & $37.1 \%$ \\
\hline Age of patients with chronic $P$. aeruginosa $(\mathrm{yrs})$ & $26.44+10.33$ & $27.67 \pm 10.18$ & $16.24 \pm 8.13$ \\
\hline Liver damage & $9.1 \%$ & $6.4 \%$ & $33.3 \%$ \\
\hline
\end{tabular}


L138ins, is an ACT insertion between nucleotides 412 and 413 , which leads to the insertion of histidine between two leucines located at 137 and 138 (c.412_413insACT; p.Leu137_Leu138insHis). In another case, the 546insCTA (c.414_415insCTA) mutation was described, however, the wrong amino acid sequence at the protein level was reported as p.Leu139X (i.e., a premature stop codon at position 139); the correct designation of this mutation should be p.Leu138dup (i.e., leucine duplication at position 138).

Since $31^{\text {st }}$ August 2018, the mutation understudy was included in the CFTR2 database, referred to as c.413_415dupTAC (p.Leu138dup, L138ins, rs397508679) [9]. It is also included in the EXAC database of 1000 genomes [10] and the ClinVar [11]. The first reference identified was the correct description of the mutation in the work of McGinniss et al. (2011) [12].

In our previous paper regarding the genotype-phenotype correlation in Russian CF patients with c.411_412insCTA (p.Leu138dup) mutation, we showed that the p.Leu138dup mutation could be considered as a disease-causing mutation, leading to a variable but relatively mild course of CF (Table 1) [13].

To date, in the Laboratory of Genetic Epidemiology, mutation L138ins (c.411_412insCTA, p.Leu138dup), in trans with other CF-causing mutations, has been detected in 37 Russian CF patients from 33 families. The average patient age is 13.13 \pm 11.42 years (1.00-43.00), with a ratio by sex: 0.43 $\mathrm{m}: 0.57 \mathrm{f}$ (16:21). Eleven different genotypes have been identified and are described in Table 2 .

The most common genotype, L138ins/ F508del (c.[411_412insCTA];[1521_1523delCTT], p.[Leu138dup];[Phe508del]), has been found in $15 \mathrm{CF}$ patients (40.5\%); the second allele remains unidentified in four patients. The L138ins (c.411_412insCTA, p.Leu138dup) mutation is always linked to one haplotype of the intrinsic marker IVS1CA-IVS6aGATT-IVS8CA-IVS17-

Table 2. Genotypes of the examined patients

\begin{tabular}{lc}
\multicolumn{1}{c}{ Genotype } & Number of patients \\
\hline L138ins/F508del (c.[411_412insCTA];[1521_1523delCTT], p.[Leu138dup];[Phe508del]) & 15 \\
\hline L138ins/CFTRdele2,3 (c.[411_412insCTA];[54-5940_273+10250del21kb], p.[Leu138dup];[Ser18Arg*fsX16]) & 4 \\
\hline L138ins/2184insA (c.[411_412insCTA];[2052_2053insA], p.[Leu138dup];Gln685ThrfsX4]) & 3 \\
\hline L138ins/2143delT (c.[411_412insCTA];[2012delT], p.[Leu138dup];[Leu671X]) & 1 \\
\hline L138ins/N1303K (c.[411_412insCTA];[3909C>G], p.[Leu138dup];[Asn1303Lys]) & 1 \\
\hline L138ins/R1162X (c.[411_412insCTA];[3484C>T], p.[Leu138dup];[Arg1162X]) & 4 \\
\hline L138ins/712-1G>T (c.[411_412insCTA];[580-1G>T] & 1 \\
\hline L138ins/E92K (c.[411_412insCTA];[274G>A], p.[Leu138dup];[Glu92Lys]) & 1 \\
\hline L138ins/2183AA->G (c.[411_412insCTA];[2051_2052delAAinsG], p.[Leu138dup];[Lys684SerfsX38]) & 1 \\
\hline L138ins/L138ins (c.[411_412insCTA];[411_412insCTA], p.[Leu138dup];[Leu138dup]) & 1 \\
\hline L138ins/W1282R (c.[411_412insCTA];[3844T>C], p.[Leu138dup];[Trp1282Arg]) & 1 \\
\hline L138ins/not identified (c.[411_412insCTA];[?], p.[Leu138dup];[?]) & 4
\end{tabular}

Table 3. Distribution of the L138ins (c.411_412insCTA, p.Leu138dup) mutation in various regions of the Russian Federation

\begin{tabular}{lcc} 
Federal regions & $\begin{array}{c}\text { The number of patients tested in the } \\
\text { laboratory of genetic epidemiology }\end{array}$ & $\begin{array}{c}\text { The proportion (\%) of the total number of } \\
\text { mutant alleles (based on the RCF-PR 2016) }\end{array}$ \\
\hline Central & 8 & 1.30 \\
\hline \multicolumn{1}{c}{ Moscow } & 16 & 1.52 \\
\hline North-Western & & $<1.00$ \\
\hline \multicolumn{1}{r}{ St. Petersburg } & & $<1.00$ \\
\hline Southern & 2 & $<1.00$ \\
\hline Volga Region & & 1.38 \\
\hline Ural & 2 & 3.00 \\
\hline Siberian & & $<1.00$ \\
\hline Far Eastern & & - \\
\hline North Caucasus & & - \\
\hline
\end{tabular}


bCA - 22-7-16-13, suggesting that the L138ins (c.411_412insCTA, p.Leu138dup) variant occurred as the result of a single mutation event.

Most CF patients with the L138ins (c.411_412insCTA, p.Leu138dup) mutation reside in Moscow (17) or the Moscow region (7) and all patients belonged to a Slavic ethnic group (Russians, Ukrainians). The L138ins (c.411_412insCTA, p.Leu138dup) mutation has been detected in six of the nine Federal Regions (predominantly Russian) of the Russian Federation, with a relative proportion ranging from $0.35 \%$ to $3.11 \%$. Relative frequencies of the L138ins (c.411_412insCTA, p.Leu138dup) mutation (according to RCF-PR 2017) are given in Table 3 [2]

\section{Conclusions}

The L138ins (c.411_412insCTA, p.Leu138dup) mutation identified in Russian CF patients (a Slavic ethnic group) is a duplication of three nucleotides (CTA) in exon 4 of the CFTR gene and is categorised as a small in-frame insertion/deletion.

\section{Acknowledgements}

\section{Conflict of interest statement}

The authors declare no conflict of interest.

\section{Funding sources}

The work was carried within the state assignment of Ministry of Science and Higher Education of the Russian Federation for Medical Genetics and in part with the financial support of the Russian Fund for Fundamental Research (RFFR) (project No. 20-015-00061) (expeditions, DNA research, writing the manuscript) and Czech Ministry of Health CZ.2.16/3.1.00/24022OPPK; IP00064203/6003 for University Hospital Motol (general management of the project, reviewing the manuscript).

\section{References}

1. Kapranov N, Kashirskaya N, eds. Cystic fibrosis (Mucoviscidosis). Moscow: Medpractika-M; 2014.

2. Krasovsky S, Chernyak A, Voronkova A, Amelina E, Kashirskaya N, Kondratyeva E, Gembitskaya T, eds.
Cystic Fibrosis Patients Registry in Russian Federation. 2016. Moscow: Medpractika-M; 2018.

3. Petrova N, Kondratyeva E, Krasovsky S, Polyakov A, Ivachshenko T, Pavlov A, Zinchenko R, Ginter E, Kutsev S, Odinokova O, Nazarenko L, Kapranov N, Amelina E, Asherova I, Gembitskaya T, Ilyenkova N, Karimova I, Merzlova N, Namazova-Baranova L, Neretina A, Nikonova V, Orlov A, Protasova T, Semykin S, Sergienko D, Simonova O, Shabalova L, Kashirskaya N. National Consensus Project «Cystic fibrosis: definition, diagnostic criteria, treatment" Section «Genetics of Cystic Fibrosis. Molecular genetic diagnosis of cystic fibrosis». Medical Genetics. 2016;15(11):29-45.

4. Petrova N. Analysis of four polymorphisms in CFTR gene in families of cystic fibrosis patients. Medical Genetics. Medical Genetics. 2006;5(12):27-32.

5. Petrova N, Timkovskaya E, Zinchenko R, Ginter E. Analysis of the frequency of some mutations in the CFTR gene in different populations of Russia. Medical Genetics. 2006;5(12):32-9.

6. Rukavichkin DV. Clinico-genotypic polymorphism of cystic fibrosis among the population of the Krasnodar Territory. Krasnodar: Diss. Cand. Med. Sciences: 03.00.15; 2007

7. Dörk T, Dworniczak B, Aulehla-Scholz C, Wieczorek D, Böhm I, Mayerova A, Seydewitz HH, Nieschlag E, Meschede D, Horst J, Pander H, Sperling H, Ratjen F, Passarge E, Schmidtke J, Stuhrmann M. Distinct spectrum of CFTR gene mutations in congenital absence of vas deferens. Human Genetics. 1997 Aug 4;100(3-4):365-377. https://doi.org/10.1007/ s004390050518

8. Cystic Fibrosis Mutation Database. http://www. genet.sickkids.on.ca. Accessed 2019 August 20.

9. Clinical and Functional Translation of CFTR. https:// www.cftr2.org. Accessed 2019 August 20.

10. Exome Aggregation Consortium. http://exac.broadinstitute.org. Accessed 2019 August 20.

11. ClinVar; [VCV000053905.2]. https://www.ncbi.nlm. nih.gov/clinvar/variation/VCV000053905.2. Accessed 2020 March 2.

12. McGinniss MJ, Buller AM, Quan F, Peng $M$, Sun W. Cystic Fibrosis Gene Mutations. 2011 Dec 13; US008076078B2 (United States).

13. Petrova N, Kashirskaya N, Vasilyeva T, Voronkova A, Kondratieva E, Sherman V, Novoselova O, Krasovskiy S, Chernyak A, Amelina E, Ginter E, Kutsev S, Zinchenko $R$. Phenotypic features in patients with cystic fibrosis with L138ins (p.Leu138dup) mutation. Pediatria. Journal named after G.N. Speransky. 2017 Dec 11;96(6):64-72. https://doi.org/10.24110/0031403x-2017-96-6-64-72 